\title{
INFORMES*
}

\section{El marco normativo andaluz de la planificación de emergencias ante el riesgo de contaminación del litoral ${ }^{1}$}

I.- El Boletín Oficial de la Junta de Andalucía núm. 130, de 2 de julio de 2008, publica el Acuerdo de 10 de junio, del Consejo de Gobierno, por el que se aprueba el Plan de Emergencia ante el Riesgo de Contaminación del Litoral en Andalucía.

Con este Plan, la Comunidad Autónoma perfila la respuesta institucional de la Junta de Andalucía a los graves problemas que plantea la contaminación del litoral, sin duda una materia de singular importancia en nuestro territorio por las circunstancias que en él concurren, que lo hacen especialmente vulnerable. En efecto, como recuerdan las normas y acuerdos aprobados, son factores clave: 1) la extensión de la costa andaluza, con casi 1100 kilómetros de longitud, de los cuáles más de 300 kilómetros forman parte de la Red de Espacios Naturales Protegidos de Andalucía; 2) la densidad del tráfico marítimo que se sucede frente a la misma; 3) la peligrosidad de algunas de las mercancías que se transportan; 4) la importante actividad económica asociada al litoral (pesca, comercio, acuicultura, turismo, puertos y establecimientos industriales); 5) los núcleos urbanos situados en el litoral.

El propósito de las páginas que siguen es el de ofrecer una panorámica general del marco normativo andaluz que regula la planificación ante el riesgo de contaminación del litoral así como, también, el de exponer el complejo organizativo que articula la planificación aprobada en esta materia.

Con carácter previo, conviene precisar que la planificación que se regula es la que concierne a las actuaciones en tierra, pues las actuaciones en mar son

\footnotetext{
* Sección redactada bajo la dirección de José Ignacio MORILLO-VELARDE PÉREZ.

${ }^{1}$ Realizado por $\mathrm{M}^{\mathrm{a}}$ del Carmen NÚÑEZ LOZANO.
} 
de la competencia de la Administración del Estado. Sin embargo, la contaminación afecta normalmente tanto al ámbito terrestre como al ámbito marítimo, de modo que se hacen precisos mecanismos de coordinación, según más adelante veremos.

II.- La norma de cabecera que regula la respuesta administrativa en Andalucía frente a los riesgos de contaminación del litoral es la Ley 2/2002, de 11 de noviembre, de Gestión de Emergencias en Andalucía (LGEA).

Su objeto es "la regulación de la gestión de emergencias en Andalucía, entendida como conjunto de acciones de las personas físicas o jurídicas, públicas o privadas, dirigidas a la protección de la vida e integridad de las personas y los bienes, en situaciones de grave riesgo colectivo, catástrofes y calamidades públicas, así como en aquellas otras situaciones no catastróficas que requieran actuaciones de carácter multisectorial y la adopción de especiales medidas de coordinación de los servicios operativos" (art. 1.1).

La Ley hace descansar la gestión de las emergencias en el establecimiento, por las Administraciones Públicas, de "un sistema integrado que dé respuesta a una efectiva coordinación, dirección y control de las actuaciones necesarias, basado en la colaboración entre las mismas y, en su caso, con entidades de carácter privado y la ciudadanía en general" (art. 1.2). Las actuaciones que deben llevar a cabo, formuladas en la LGEA como principios, son: 1) previsión de riesgos, orientada a una adecuada labor de planificación, mediante técnicas de identificación y análisis; 2) reducción de riesgos, mediante una adecuada política de prevención, adopción de medidas correctoras, actividad de inspección y sanción; 3) elaboración y aprobación de planes de emergencia y protocolos operativos; 4) adopción de medidas de intervención destinadas a paliar en lo posible las consecuencias de los eventos producidos; 5) establecimiento de programas de rehabilitación; 6) formación de los ciudadanos que puedan resultar afectados por las situaciones de emergencia, la información a los mismos, así como la capacitación y reciclaje de los técnicos de protección civil y personal de los servicios intervinientes (art. 3).

En particular por lo que concierne a la planificación, el art. 7 LGEA la caracteriza como una actuación básica ante situaciones de grave riesgo, catástrofe o calamidad pública. Y el art. 10 se refiere a los planes de emergencia como "el instrumento normativo mediante el que se establece el marco orgánico y funcional, así como los mecanismos de actuación y coordinación, ante situaciones de grave riesgo, catástrofe o calamidad pública”, estableciendo la siguiente tipología: 1) planes territoriales de emergencia; 2) planes especiales 
y planes específicos; 3) planes de emergencia interior o de autoprotección; 4) planes sectoriales.

De entre ellos, nos interesan los planes específicos de emergencia, que son "aquellos $[\mathrm{sic}]$ elaborados para hacer frente a las emergencias generadas por riesgos de especial significación en Andalucía, cuya naturaleza requiera una metodología técnico-científica adecuada para ello, y así haya sido apreciada por el Consejo de Gobierno de la Junta de Andalucía, mediante la aprobación de la correspondiente norma marco, que, en todo caso, establecerá el contenido mínimo a que deberán adaptarse los correspondientes planes específicos de emergencia" (art. 13.2).

Tales requisitos se cumplen en la planificación que nos ocupa, y así lo apreció el Consejo de Gobierno en su Acuerdo de 23 de septiembre de 2003, por el que se aprueba la Norma Marco de Planificación de Emergencia ante Contaminación del Litoral, de la que nos ocuparemos a continuación ${ }^{2}$.

III.-El Acuerdo del Consejo de Gobierno de 23 de septiembre de 2003, por el que se aprueba la Norma Marco de Planificación de Emergencia ante Contaminación del Litoral, es consecuencia, en efecto, de la constatación de la vulnerabilidad de este espacio; del convencimiento de que "la actuación ante potenciales siniestros con consecuencias catastróficas en el litoral andaluz precisa desarrollar herramientas de gestión de emergencias eficaces y adecuadas", así como la "aplicación de los principios de prevención y planificación" (preámbulo del Acuerdo); y de la apreciación de que "la naturaleza de dicho riesgo requiere una metodología técnico-científica adecuada".

A través de la Norma Marco se establecen los elementos básicos para la planificación y su estructura general; en este sentido, y como ella afirma al trazar su objeto, establece los requisitos mínimos de los planes. Asimismo, se detalla lo concerniente a la aprobación de los planes, la determinación de las emergencias y la integración con la planificación sectorial.

Los elementos básicos para la planificación son las tipologías de riesgos, el análisis de riesgos y zonificación territorial y los sistemas de previsión de

\footnotetext{
${ }^{2}$ No hemos encontrado la publicación de este Acuerdo en el BOJA. Puede consultarse en http://www.proteccioncivil-andalucia.org/Legislacion/Riesgos/ContAmbiental/ACU23SEPT20 03ContAmbiental.pdf
} 
peligros. Por lo que concierne a la tipología de riesgos, la Norma Marco prescribe que se consideren todas las causas que puedan ser origen de la contaminación producida por una instalación mar adentro, un puerto, terminal marítimo o industria del litoral, así como de la contaminación producida por un accidente marítimo en el que estén involucrados uno o más buques, tal como una colisión, una varada o avería. Por lo que respecta a la caracterización del riesgo, se deben considerar al menos las siguientes cuestiones: 1) caracterización de las zonas costeras; 2) análisis de sectores, actividades e instalaciones generadoras de riesgo; 3) estudios de peligrosidad y probabilidad; 4) identificación y valoración de vulnerabilidad; 5) determinación de mapas de riesgo. Por último, los sistemas de previsión de peligros deben proporcionar, ante una situación de vertido o derrame, la información necesaria sobre la situación que pueda producir la contaminación originada, así como la evaluación de las circunstancias, con objeto que puedan adoptarse medios adecuados de protección de personas, medio ambiente y bienes y alertar a la población que pueda resultar afectada.

La Norma Marco prevé distintos planes que han de conformarse como "parte de una estructura integral capaz de hacer frente de forma ágil y coordinada a los distintos supuestos que puedan presentarse" ${ }^{3}$. Tales planes son: 1) el Plan de Comunidad Autónoma; 2) los planes de actuación local; 3) los planes interiores o de autoprotección.

El Plan de Comunidad Autónoma es el Plan de Emergencia ante el Riesgo de Contaminación del Litoral. Su elaboración corresponde a la Junta de Andalucía y en el mismo se pueden establecer dos niveles, regional y provincial, en función del carácter de la emergencia y del territorio afectado.

Los objetivos que han de conseguirse con el Plan son: 1) disponer medidas y herramientas de prevención de riesgos frente a la contaminación desde el mar al litoral; 2) prever un dispositivo de intervención inmediata ante situaciones de emergencia, 3) establecer órganos e instrumentos para la activación, seguimiento, coordinación y dirección de las actuaciones en emergencias y post-emergencias; 4) desarrollar técnicas de intervención que no

\footnotetext{
${ }^{3}$ Añade la Norma Marco que "para ello, resulta necesario que en la planificación a los distintos niveles se establezcan órganos y procedimientos que hagan posible la integración en un conjunto plenamente operativo y susceptible de una rápida aplicación, así como prever las relaciones funcionales precisas entre los Planes a distinto nivel, al objeto de facilitar la coordinación y asistencia mutua entre los mismos en el caso que sea necesario".
} 
supongan riesgos para la recuperación de las zonas afectadas; 5) disponer las medidas de rehabilitación de carácter urgente, 6) dar a conocer las medidas de emergencia mediante programas de formación, información y divulgación, tanto a los intervinientes como a la población.

A tal efecto, la Norma Marco enumera los aspectos a que debe atender la estructura y organización del Plan: 1) definir los órganos dirección y coordinación; 2) establecer el esquema de relación entre los diversos servicios, organismos y entidades implicadas en la adopción de medidas; 3) disponer un servicio de intervención inmediata capaz dar una respuesta rápida ante emergencias, en alerta permanente las 24 horas, todos los días del año; 4) prever un marco de asesoramiento técnico-científico que estudie de forma continua las mejoras y opciones de lucha contra la contaminación en los supuestos relativos al Plan; 5) articular la participación de colectivos que pueden contribuir en la mitigación de los efectos; 6) promover la mayor coordinación entre las diferentes Administraciones Públicas competentes; 7) garantizar la integración del Plan en el marco del Plan Territorial del Emergencias de Andalucía ${ }^{4}$.

La Norma Marco señala de antemano cuáles son los órganos de dirección, coordinación e información, así como sus funciones básicas, de manera que la tarea que deja para el Plan es, en principio, la de determinar la composición concreta de los órganos, como indica el apartado 3.3.3.1 de la Norma Marco. Tales órganos de dirección, coordinación e información son: 1) la Dirección del Plan; 2) el Comité Asesor; 3) el Gabinete de Información; 4) el Comité Técnico-Científico; 5) el Órgano de Participación; 6) el Comité de Operaciones. También establece el perfil y las funciones del Centro de Coordinación Operativa (CECOP) y del Puesto de Mando Avanzado (PMA), que integran el Centro de Operaciones ${ }^{5}$. Igualmente define los Grupos Operativos y establece cinco de ellos como referencia (de Intervención, de Seguimiento, Sanitario, de Seguridad y de Apoyo Logístico), precisando las funciones básicas de cada uno de ellos. Ha de tenerse en cuenta que el Anexo

\footnotetext{
${ }^{4}$ Vid. el art. 12 LGEA. De hecho, la organización que se diseña en la Norma Marco se ajusta a la que se establece en el Plan Territorial de Emergencias de Andalucía (aprobado por el Consejo de Gobierno en sesión de 6 de octubre de 1998 y cuya publicación aprueba el Acuerdo del Consejo de Gobierno de 13 de octubre de 1999; vid. BOJA núm. 130, de 9 de noviembre de 1999). La integración es especialmente visible en el Centro de Coordinación Operativa, como seguidamente se verá.
}

${ }^{5}$ El CECOP es la configuración que adquiere el Centro de Coordinación de Emergencias (art. 23 LGEA) cuando se activa el Plan. 
III del Acuerdo del Consejo de Gobierno incorpora propuestas para la composición de los órganos.

La operatividad se aborda en la Norma Marco mediante la caracterización de las fases de emergencia y la descripción de las líneas básicas del procedimiento operativo, ofreciendo directrices precisas en la materia en orden a la elaboración del Plan. También se contempla la implantación y mantenimiento del Plan, así como cuestiones referidas a la investigación y desarrollo. Sobre estas últimas, prevé que el Plan impulse la creación de un registro de emergencias por contaminación marina y costera que facilite el estudio y mejora de las técnicas; la creación de una base de datos con las emergencias producidas por vertidos o derrames; $y$, asimismo, que se apoyen las actuaciones de investigación y desarrollo de técnicas de lucha, descontaminación y recuperación de áreas afectadas por catástrofes ambientales, la elaboración de guías y documentos de apoyo a la gestión de emergencias ambientales relacionados con la prevención y las primeras medidas paliativas y la identificación de las necesidades materiales y nuevos equipamientos para la lucha contra la contaminación.

Menos prolija es la Norma Marco en lo se refiere a los planes de actuación local y a los planes de autoprotección. Los primeros han de ser elaborados por los municipios que se sitúen en las zonas de riesgo por contaminación del litoral; los segundos por los titulares de instalaciones mar a adentro, puertos, terminales marítimos o industrias del litoral. De unos y otros se señala que se integran en el Plan de Comunidad Autónoma a través de la estructura provincial y se precisa su contenido mínimo.

Se regulan además diversos extremos atinentes a la aprobación de los planes, tanto el Plan de la Comunidad Autónoma como los planes de actuación local.

La Norma Marco contempla como supuesto especial la declaración de emergencia de interés general de Andalucía, en desarrollo del art. 16.2 LGEA, que implica la articulación de un dispositivo singular.

Por último, se ocupa de la integración de la planificación sectorial cuyo contenido sea susceptible de afectar a la materia: los instrumentos correspondientes habrán de sujetarse a los criterios de la Norma Marco y a la legislación vigente; previamente a su aprobación por el órgano competente, los planes sectoriales deberán ser sometidos a la consideración de la Comisión de Protección Civil de Andalucía, que informará sobre su adaptación a la norma- 
tiva vigente sobre contaminación del litoral y gestión de emergencias, así como sobre su adaptación a las directrices y criterios contenidos en el Plan Territorial de Emergencia de Andalucía, en la Norma Marco así como en el Plan de Emergencia ante la Contaminación del Litoral de la Comunidad Autónoma de Andalucía.

IV.-Finalmente, el Acuerdo de 10 de junio de 2008, que ha motivado estas páginas, aprueba el Plan de Emergencia ante el Riesgo de Contaminación del Litoral en Andalucía (PECLA es el acrónimo que recibe en el Acuerdo).

El objeto fundamental del PECLA es "el establecimiento de la estructura organizativa y de los procedimientos de actuación para una adecuada respuesta ante situaciones de emergencia en las que se origine contaminación en la Comunidad Autónoma de Andalucía, asegurando una mayor eficacia y coordinación en la intervención de los medios y recursos disponibles". Con el Plan se pretende ofrecer una respuesta "integral y coordinada" y, asimismo, "organizada", que integre los medios de la Comunidad Autónoma y, también, los que asignen otras Administraciones Públicas y, en general, Entidades públicas y privadas. En este sentido, concreta la Norma Marco en lo que concierne a la planificación que compete a la Comunidad Autónoma.

Son varios los aspectos del PECLA que merecen ser destacados.

En primer término, presupone un análisis y evaluación del riesgo que, según se dice en el propio Plan, ha llevado a cabo la Consejería de Gobernación. También se indica en el Plan que se ha realizado una zonificación del riesgo, atendiendo a los factores de vulnerabilidad, peligrosidad y exposición y considerando las diversas causas accidentales que pueden ser origen de la contaminación. La finalidad del análisis ha sido la de posibilitar el establecimiento de hipótesis de accidentes y de posibles respuestas. El mismo, empero, se ha centrado especialmente en los riesgos derivados de accidentes de tráfico marítimo relacionados con el transporte de hidrocarburos, lo que se ha justificado en las circunstancias de ser éstos los de mayor relevancia y magnitud de impacto ${ }^{6}$. En el PECLA no queda reflejado el análisis y

\footnotetext{
${ }^{6}$ Se expresa en el PECLA que "se ha tenido en cuenta que los hidrocarburos son los productos de mayor representatividad en el transporte marítimo, frente a otras sustancias como fertilizantes, productos químicos, residuos, etc. Así mismo, el impacto ecológico y socioeconómico de este tipo de vertidos en el litoral suele ser significativamente mayor que el de aquellos producidos por accidentes en instalaciones e industrias”.
} 
la evaluación del riesgo ${ }^{7}$, aunque sí se describe la metodología seguida, encaminada a la obtención de dos valores, uno aplicado al riesgo socioeconómico y otro aplicado al riesgo ecológico. La información generada se ha plasmado en una cartografía temática, útil para obtener una visión espacial de las posibles consecuencias de un vertido y para realizar previsiones sobre medios y recursos necesarios para la prevención e intervención, tanto en la zona en cuestión como en otras colindantes o de apoyo ${ }^{8}$.

En segundo lugar, determina la composición de los órganos, estructuras y unidades contemplados en el Acuerdo-Marco. Deben subrayarse los siguientes aspectos: 1) atribuye, con mejor técnica, la Dirección del Plan, a nivel regional, a la persona titular de la Consejería competente en materia de protección civil (en la Norma Marco corresponde a la persona titular de la Consejería de Gobernación); 2) opta por una configuración amplia del Comité Asesor, en el que se integran representantes de las distintas Administraciones Públicas y, específicamente, de órganos y Entidades con competencias sectoriales relevantes ${ }^{9}$; 3) por lo que concierne al Comité Técnico-Científico y al Órgano de Participación, asigna a la Dirección Regional del Plan la determinación concreta de sus integrantes, si bien señala referencias que han de guiar la designación; 4) abunda en la descripción de los Centros de Coordinación de Emergencia, que son los que se configuran como CECOP tras la activación del plan ${ }^{10} \mathrm{y}$, asimismo, recoge la consideración del CECOP como centro de coordinación operativa integrado en las situa-

\footnotetext{
${ }^{7}$ Puede dudarse si el PECLA debía o no incluir la información obtenida en el análisis de riesgo y zonificación territorial. Pero la Norma Marco es taxativa cuando indica que "se incluirá dentro del Plan el listado de industrias o instalaciones susceptibles de riesgo, con la correspondiente localización en el territorio".

${ }^{8}$ Recogemos aquí las expresiones que utiliza el PECLA. Tal cartografía no se ha publicado.

${ }^{9}$ Por ejemplo, Fuerzas y Cuerpos de Seguridad, Instituto Nacional de Meteorología, Puertos del Estado, Dirección General de Marina Mercante o Dirección General de Costas. Hubiera sido más correcta la referencia al órgano o Entidad por su competencia, en vez de la cita nominal.

${ }^{10}$ El PECLA reseña al respecto que el Centro de Coordinación de Emergencias-112 Andalucía (CECEM-112 Andalucía) constituye un sistema en red con instalaciones en todas las provincias andaluzas. Presta servicio de forma continuada e ininterrumpida veinticuatro horas todos los días del año. Dispone de una amplia infraestructura de comunicaciones que le permite la interconexión y complementariedad entre las distintas instalaciones provinciales. Tiene una actividad permanente dirigida a la recepción de avisos, solicitudes e informaciones, así como a transmitir información a distintos organismos y servicios.
} 
ciones en las que en éste intervengan efectivos de las distintas Administraciones Públicas implicadas; 5) deja abierta la posibilidad de incorporar a los Grupos Operativos nuevos efectivos, en función de la evolución de la emergencia y de la disponibilidad de medios y recursos; 6) en términos generales, completa las propuestas del Anexo III del Acuerdo de 23 de septiembre de 2003.

Por otra parte, perfila con más de detalle las funciones de los diversos órganos y los modos de proceder. Cabe destacar que: 1) prevé la integración de la persona responsable de la Dirección Provincial del Plan en el Comité Asesor Regional si la emergencia evoluciona de nivel provincial a regional; 2) sólo se convoca al Comité Técnico-Científico y al Órgano de Participación si la emergencia es de nivel regional; si es de nivel provincial, se prevé la posibilidad de que se incorporen al Comité Asesor provincial representantes del Comité Técnico-Científico ${ }^{11}$; 3) se reasigna la función de analizar y valorar la situación de emergencia, que pasa de la Dirección del Plan al Comité Asesor; 4) excluye al Comité de Operaciones del conjunto de órganos calificados como de dirección, coordinación e información; 5) contempla el incremento de las funciones de los Grupos Operativos según el desarrollo de la emergencia.

Mención destacada merecen las previsiones de coordinación e integración entre los diversos ámbitos de planificación: planes de autoprotección, planes de actuación local y PECLA, de un lado; y, de otro, Plan Nacional de Contingencias $^{12}$ y PECLA. El PECLA acompaña, a propósito de la coordinación de las actuaciones en mar y en tierra, dos organigramas en los que se muestran la estructura de integración tanto a nivel regional como a nivel provincial, que pivota sobre la constitución de un órgano mixto, denominado "Organismo Rector" y sobre la integración en el Comité de Operaciones del coordinador de operaciones marítimas y del coordinador de operaciones terrestres $^{13}$.

\footnotetext{
${ }^{11}$ En la Norma Marco, en cambio, se señala que "en su estructuración provincial, el Órgano de participación tendrá además una vertiente operativa dirigida a regular y organizar la intervención del voluntariado".

${ }^{12}$ Aprobado por Orden comunicada del Ministerio de Fomento de 23 de febrero de 2001, que no fue publicada en el BOE. Puede consultarse el archivo escaneado en la siguiente página: http://www.ccmm-prestige.cesga.es/Resoluciones/Contingencias.htm

${ }^{13}$ En la Norma Marco sólo se prevé la constitución del Organismo Rector a nivel regional.
} 
En lo concerniente a la operatividad, el PECLA pormenoriza con sumo detalle las previsiones de la Normativa-Marco, como es natural.

Por lo que respecta a la implantación y al mantenimiento, el Plan reitera las previsiones genéricas de la Norma Marco, aunque concretando especialmente las actuaciones de información y divulgación y las de actualización; por otro lado, se establece una periodicidad de cinco años para las revisiones. El PECLA, sin embargo, omite los interesantes aspectos de investigación y desarrollo que contiene la Norma Marco.

Finalmente el PECLA, excediéndose de lo que sería su contenido natural, aborda también los Planes de Actuación Local ${ }^{14}$, estableciendo lo que ciertamente parece la normativa de desarrollo de la Normativa-Marco en la materia. Así, define su objeto; enumera sus funciones básicas; establece su contenido mínimo (que estructura en introducción, descripción del municipio y ámbito de aplicación, análisis de riesgos, estructura y organización, operatividad, implantación y mantenimiento e, incluso, anexos ${ }^{15}$ ); y regula el Centro de Coordinación Operativo Local (CECOPAL). Sí guardan coherencia con su carácter de Plan regional las previsiones sobre la interfase de los PAL y el propio PECLA.

\footnotetext{
${ }^{14}$ En cambio, apenas trata los planes de autoprotección. Indica que las instalaciones industriales y portuarias deben disponer de sus respectivos Planes de Autoprotección. Especifica después que corresponde su elaboración a los titulares de la actividad generadora de riesgo y/o susceptible de sufrir daños; que deben ser elaborados conforme a las normativas de aplicación; que serán activados en primera instancia en todas aquellas emergencias que afecten al ámbito de la correspondiente instalación o sean causa de daños en el exterior; y que su implantación y aplicación es responsabilidad del correspondiente titular. Se prescribe también su integración con las demás figuras de planificación.

${ }^{15}$ Seis en concreto: 1) Zonas y población potencialmente afectadas; 2) Procedimientos de evacuación. Rutas principales; 3) Zonas de seguridad. Áreas e instalaciones de alojamiento; 4) Catálogo de medios y recursos; 5) Relación de empresas obligadas a elaborar plan de autoprotección, 6) Cartografía.
} 